\title{
Renal Activity of Akt Kinase in Experimental Type 1 Diabetes
}

\author{
J. ŽDYCHOVÁ, J. VESELÁ, L. KAZDOVÁ, R. KOMERS \\ Diabetes Center, Institute for Clinical and Experimental Medicine, Prague, Czech Republic
}

Received June 29, 2007

Accepted August 9, 2007

On-line October 11, 2007

\begin{abstract}
Summary
Akt kinase regulates numerous cell functions including glucose metabolism, cell growth, survival, protein synthesis, and control of local hemodynamics. mTOR is one of down-stream effectors of Akt involved in the initiation of protein translation. However, renal Akt signaling in Type 1 diabetes (DM) in vivo, in particular under the conditions reflecting differences in metabolic control, has received less attention. Renal cortical activity and expression of Akt and mTOR (kinase assay, western blotting) were determined in streptozotocin-diabetic rats (D) with different levels of glycemic control (blood glucose 22.0 $\pm 1.0,13.4 \pm 1.5$, $8.1 \pm 0.4 \mathrm{mmol} / \mathrm{l}, \mathrm{p}<0.05$ between the groups), achieved by varying insulin treatment (0, 4 and $12 \mathrm{IU} /$ day), and in control rats with (C4) or without (C) chronic insulin administration. Renal Akt activity was reduced in $\mathrm{D}$ rats without insulin treatment and severe hyperglycemia (D-0, $-62 \%, p<0.01$ vs. C), partially restored in moderately hyperglycemic rats (D-4, $-30 \%, p<0.05$ vs. C), and normalized in $D$ rats with intensive insulin and tight metabolic control (D-12). Expression of active mTOR paralleled Akt activity in D-0 (-51\%, p $<0.01$ vs. C), but not in D-4 and D12 that demonstrated increases in active mTOR $(+55 \%,+80 \%$ resp., $\mathrm{p}<0.05)$ as compared to $\mathrm{C}$. Moreover, insulin activated renal Akt $(+82 \%, \mathrm{p}<0.01)$, but not mTOR in C4. In conclusion, glycemic control and intensity of insulin treatment are important modulators of renal Akt and mTOR activity in diabetes. While Akt activity is reversible by tight metabolic control, combination of hyperglycemia and insulin treatment resulted in enhancement of mTOR activity. In addition to Akt, other signaling pathways likely contribute to regulation of renal mTOR activity in diabetes.
\end{abstract}

\section{Key words}

Akt kinase • Diabetic nephropathy • Insulin • Mammalian target of rapamycin $\bullet \mathrm{mTOR}$

\section{Corresponding author}

Radko Komers, Diabetes Center, Institute for Clinical and Experimental Medicine, Videnska 1958, Prague, Czech Republic. Fax: 4202-26136-3183. E-mail: radko.komers@medicon.cz.

\section{Introduction}

Akt/protein kinase $\mathrm{B}(\mathrm{Akt})$ has been implicated in regulation of a number of cell functions including glucose uptake, glycogen synthesis, cell growth, survival, apoptosis, protein synthesis, and endothelial nitric oxide production (Shiojima and Walsh 2002). Akt can be activated by a wide variety of growth stimuli, such as growth factors and cytokines, and represents an important intermediate in insulin signaling (Downward 1998). Akt activation typically occurs via tyrosine kinase receptors such as receptors for insulin or growth factors in an phosphatidylinositol 3 - kinase (PI3K)-dependent manner (Downward 1998). Mammalian target of rapamycin (mTOR) is one of the down-stream targets of Akt involved in regulation of protein synthesis. Upon insulin and growth factor stimulation, mTOR is phosphorylated (activated) by Akt (Scott et al. 1998) and facilitates protein translation through inhibitory phosphorylation eIF-4E binding protein (4E-BP1) (Bhandari et al. 2001), a translation repressor. These events result in activation of the translation of specific mRNA subpopulations.

Renal hypertrophy, extracellular matrix (ECM) accumulation, altered apoptosis as well as changes in regional hemodynamics have been implicated in the pathophysiology of nephropathy in Type 1 diabetes (DM1) (Cooper 1998). Considering multiple functions of Akt and mTOR, it is possible that alterations in activities of these molecules may play a role in pathophysiology of diabetic nephropathy. Enhanced protein synthesis may contribute to the development of renal hypertrophy via enhanced extracellular matrix accumulation, one of the key elements in the pathophysiology of diabetic nephropathy.

In DM1, the kidney is exposed to fluctuating levels of blood glucose and exogenous insulin, 
administered as the major treatment of the disease. Although it has been well established that both glucose and insulin, can modulate Akt activity in various cell types and tissues (Dobrzynski et al. 2002, el-Remessy et al. 2005, Kondo and Kahn 2004, Laviola et al. 2001, Lee et al. 2007, Mariappan et al. 2007, Sheu et al. 2004), renal Akt signaling in DM1 in vivo, in particular in clinically relevant conditions reflecting differences in metabolic control and insulin treatment, has received less attention. To address this issue, we determined Akt and mTOR activity and expression in renal cortex of STZdiabetic rats at early stages of nephropathy with different levels of glycemic control achieved by varying insulin treatment. In addition, we also studied effects of chronic insulin administration on Akt and mTOR in non-diabetic rats.

\section{Methods}

\section{Diabetic rat model}

Studies were conducted in adult male Wistar rats (Anlab, Prague, Czech Republic) with initial body weight of about $270 \mathrm{~g}$. The rats were made diabetic by intraperitoneal injection of streptozotocin (Sigma, St. Louis, MO), $65 \mathrm{mg} / \mathrm{kg}$ body weight. Three days later, induction of diabetes was confirmed by measurements of tail blood glucose (BG) level using a reflectance meter (One Touch II, Lifescan, Milpetas, CA). All experiments were carried out with the approval of, and in accordance with the regulations of, the Institutional Animal Care and Use Committee of the Institute for Clinical and Experimental Medicine. The animals were housed with a light-dark cycle of 12 hours each, and with free access to food (standard chow) and water.

\section{Study design}

Diabetic rats were then randomized to receive no insulin treatment (severely diabetic rats, DM-0, $n=6$ ), $4 \mathrm{IU}$ of insulin/day (Insulatard, Novo Nordisk, Copenhagen, Denmark) to achieve moderate hyperglycemia (DM-4, n=6), or $12 \mathrm{IU}$ of insulin/day (diabetic rats on intensive insulin treatment, DM-12, $\mathrm{n}=6$ ), to achieve tight metabolic control. Age-matched non-diabetic rats served as controls $(C, n=8)$. In addition, non-diabetic rats treated with 4 IU of insulin/day (C4, $n=5$ ) were also studied. After 4 weeks of diabetes, the rats were sacrificed, and the blood was collected for determinations of blood glucose and plasma insulin levels. The kidneys were quickly removed on ice, divided into cortical and medullary portions, homogenized in lysis buffer as previously described (Komers et al. 2007). Aliquotes were frozen in liquid nitrogen and stored in $-70{ }^{\circ} \mathrm{C}$. Total protein concentration in each sample was determined by BCA analysis (Pierce Biotechnology, Inc. Rockford, IL).

\section{Akt kinase assay}

The homogenates were analyzed using Akt kinase activity assay kit (Cell Signaling, MA) according to the manufacturer's instructions. In brief, renal cortical homogenates $(300 \mu \mathrm{g})$ were immunoprecipitated with immobilized anti-phospho-Akt antibody and incubated with the Akt substrate GSK-3 in the presence of ATP. Protein expression of phosphorylated GSK-3, as a measure of Akt activity, was then determined by western blotting using the anti-phospho-GSK-3 antibody.

\section{Immunoblot analysis}

Equal amounts of protein of each sample were analyzed by western blotting and visualized as previously described (Komers et al. 2007) using primary antibodies raised against phospho-Serine-473-Akt (P-Ser473-Akt, 1:500, Cell Signaling, Beverly, MA) or phospho-Serine2448-mTOR (P-Ser2448-mTOR, 1:500, Cell Signaling). Following the detections of phosphoproteins, total Akt and total mTOR proteins were detected on stripped membranes. To determine equality of loading, membranes were restripped and reprobed for actin (Santa Cruz, Santa Cruz, CA).

\section{Analytical methods}

Serum insulin concentrations were measured using a rat insulin RIA kit (Amersham Biosciences, Piscataway, NJ).

\section{Statistical analysis}

Data are expressed as mean \pm SEM. All analyses were performed by analysis of variance (ANOVA) followed by the Scheffé test. $\mathrm{P}<0.05$ value was considered as statistically significant.

\section{Results}

Physical and metabolic parameters in control and diabetic rats reflected approximately differences in metabolic control (Table 1). DM-0 and DM-4 demonstrated reduced weight gain, as compared to control animals and diabetic rats with insulin treatment. 
Table 1. Metabolic and hormonal characteristics in and control and diabetic rats

\begin{tabular}{lcccccc}
\hline & $\mathbf{n}$ & $\mathbf{B W}$ & $\mathbf{R K W}$ & $\mathbf{R K W} / \mathbf{1 0 0} \mathbf{g} \mathbf{B W}$ & $\begin{array}{c}\text { Plasma insulin } \\
\mathbf{( n m o l} / \mathbf{l})\end{array}$ & $\begin{array}{c}\text { Blood glucose } \\
(\mathbf{m m o l} / \mathbf{l})\end{array}$ \\
\hline Control & 6 & $335 \pm 10$ & $1082 \pm 22$ & $0.33 \pm 0.01$ & $1.03 \pm 0.14$ & $5.4 \pm 0.1$ \\
$D M-0$ & 6 & $270 \pm 11 \mathrm{~b}$ & $1338 \pm 50 \mathrm{~b}$ & $0.50 \pm 0.01 \mathrm{~b}$ & $0.15 \pm 0.03 \mathrm{~b}$ & $22.0 \pm 1.0 \mathrm{~b}$ \\
$D M-4$ & 6 & $309 \pm 6 \mathrm{ad}$ & $1158 \pm 20^{\mathrm{c}}$ & $0.38 \pm 0.01 \mathrm{bd}$ & $1.42 \pm 0.12 \mathrm{~d}$ & $13.4 \pm 1.5 \mathrm{bd}$ \\
$D M-12$ & 6 & $343 \pm 5 \mathrm{de}$ & $1268 \pm 36^{\mathrm{be}}$ & $0.37 \pm 0.01 \mathrm{bd}$ & $1.82 \pm 0.21 \mathrm{ad}$ & $8.1 \pm 0.4 \mathrm{bde}$ \\
$C 4$ & 5 & $345 \pm 3$ & $1056 \pm 32$ & $0.33 \pm 0.01$ & $1.41 \pm 0.14$ & $5.0 \pm 0.8$ \\
\hline
\end{tabular}

BW, body weight; RKW, right kidney weight. ${ }^{\mathrm{a}} \mathrm{p}<0.05,{ }^{\mathrm{b}} \mathrm{p}<0.01$ vs. Control; ${ }^{\mathrm{c}} \mathrm{p}<0.05,{ }^{\mathrm{d}} \mathrm{p}<0.01$ vs. DM-0; ${ }^{\mathrm{e}} \mathrm{p}<0.05$ vs. DM-4.

Body weights in DM-4 rats were greater than in DM-0 rats, but lower than in DM-12 rats. All groups of diabetic rats demonstrated renal hypertrophy assessed both by kidney weight and kidney to body weight ratios. Renal hypertrophy was most prominent in DM-0 rats that demonstrated higher kidney/body weight ratios as compared to diabetic rats with insulin treatments. All groups of diabetic rats had higher BG as compared to control animals, and there were also significant differences in BG between the groups of diabetic rats, consistent with the intensity of insulin treatment. DM-0 rats had markedly lower plasma insulin levels than other groups of rats. In DM-4 rats, plasma insulin was not different from controls, whereas the DM-12 rats demonstrated significant hyperinsulinemia. Control rats receiving $4 \mathrm{IU}$ of insulin displayed no statistically significant differences in physical and metabolic parameters when compared to control animals. Plasma insulin levels in $\mathrm{C} 4$ group were higher than in untreated control animals, although this difference did not reach statistical significance.

There were marked decreases in P-Ser473-Akt expression in DM-0 rats, lacking insulin treatment (Fig.1A). Treatment with suboptimal insulin dose in DM-4 rats resulted in partial restoration of Akt phosphorylation, whereas DM-12 rats demonstrated similar expression of P-Ser473-Akt as controls (Fig. 1A). Both treatments with suboptimal (DM-4) and intensive insulin (DM-12) regimen were associated with increases in Akt phosphorylation compared to DM-0 group. However, there was no statistical difference in P-Ser473Akt expression between the DM-4 and DM-12 rats despite substantial differences in insulin dose. Expression of total Akt protein, consisting of both active and inactive kinase, did not differ between groups (Fig. 1A). Similar to analysis of P-Ser473-Akt expression, in vitro kinase assay revealed significant reduction in Akt kinase activity in DM-0 as compared to both control (Fig. 1B) and D-12 rats, partial restoration of Akt activity in DM-4 and similar Akt activity in DM-12 rats as in controls.

In DM-0 rats, mTOR phosphorylation was reduced as compared to control animals (Fig. 2). In contrast to DM-0, P-Ser2448-mTOR expression in DM-4 and DM-12 rats was higher than in control animals $(p<0.05)$. Total mTOR expression in diabetic rats was not different from that in controls (Fig. 2).

Administration of insulin to normal rats was associated with increased expression of P-Ser473-Akt (Fig. 3A). Expression of total Akt protein did not change. In contrast to Akt, expression of P-Ser2448-mTOR, as well as total mTOR protein, were similar in control and C4 rats (Fig. 3B).

\section{Discussion}

In the present studies renal Akt and mTOR activity and expression were measured in diabetic rats with different levels of metabolic control achieved by complete lack or different doses of exogenous insulin. Diabetic rats with severe hyperglycemia, no insulin treatment, and markedly reduced plasma insulin levels, demonstrated marked reductions in renal cortical Akt kinase activity. Akt activity was partially restored in moderately hyperglycemic diabetic rats treated with suboptimal dose of insulin, and completely normalized in diabetic rats with intensive insulin treatment and the best glycemic control. In addition, non-diabetic rats treated with insulin that achieved similar plasma insulin levels as moderately hyperglycemic diabetic rats, displayed marked increases in Akt phosphorylation as compared to control animals without insulin treatment.

The two major components of diabetic metabolic 

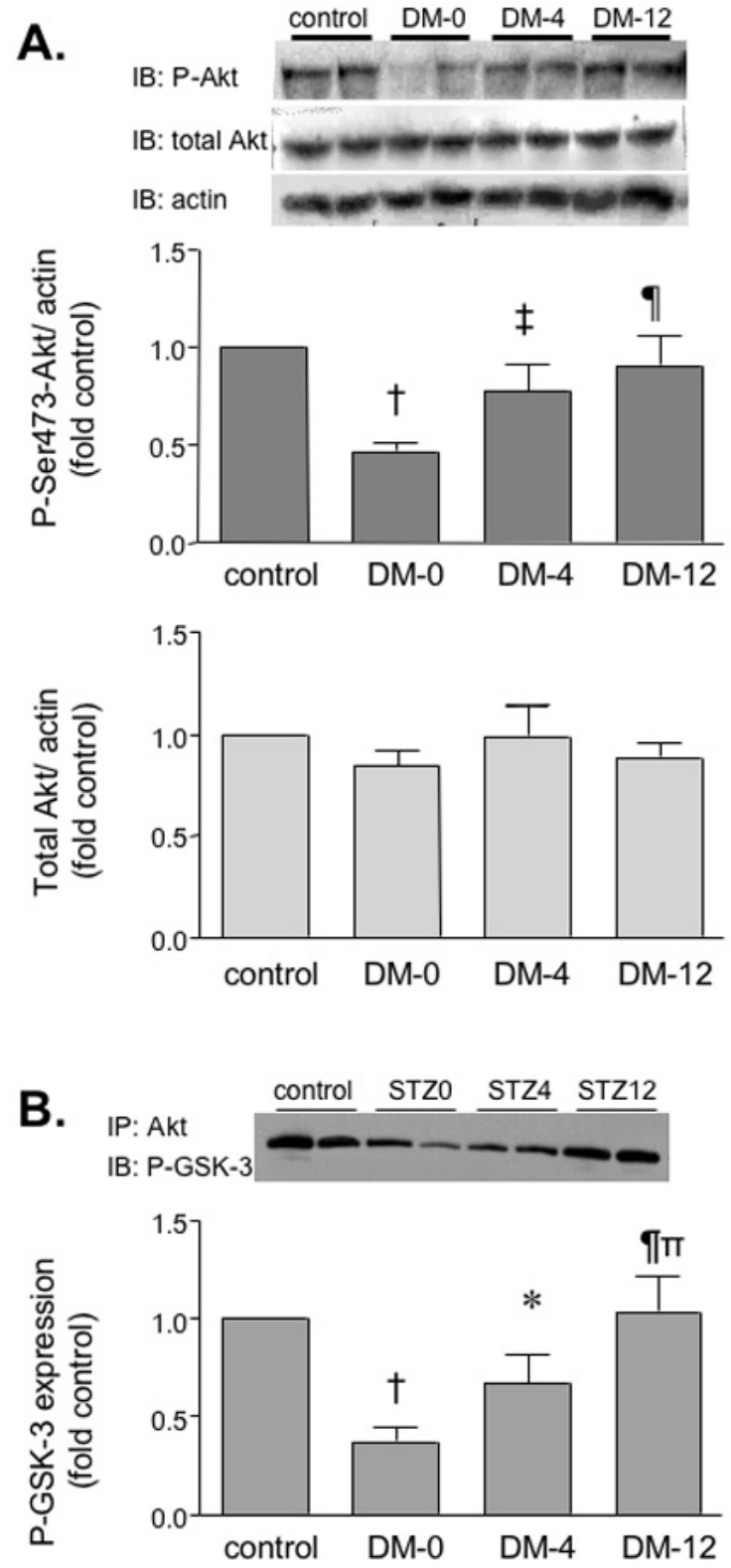

Fig. 1. Renal cortical protein expression and activity of Akt kinase in control and diabetic rats. A. P-Ser473-Akt (active) and total Akt in renal cortical homogenates harvested from control animals, diabetic rats without insulin treatment (DM-0), and diabetic rats treated with 4 IU (DM-4) and 12 IU (DM-12) of insulin as described in Methods. The data are presented as Akt/actin ratios. Representative blots are shown in the upper inset. B. Renal cortical Akt activity in control and diabetic rats determined by in vitro kinase assay using GSK-3 as a substrate. A representative blot of phospho-GSK-3 is shown in the upper panel. $* p<0.05$ vs. control; $\dagger p<0.01$ vs. control; $\neq p<0.05$ vs. DM-0; ๆ $p<0.01$ vs. DM- $0 ; \pi p<0.05$ vs. DM-4.

milieu that have been modulated in this study, i.e. glucose and insulin, have been previously shown to strongly influence Akt activities in various cell types and tissues (Dobrzynski et al. 2002, el-Remessy et al. 2005, Kondo and Kahn 2004, Laviola et al. 2001, Lee et al. 2007, Mariappan et al. 2007, Sheu et al. 2004). Our present observations also indicate that metabolic control is an important factor in the control of renal cortical Akt activity in diabetes. Akt activity in diabetic rats reflected variations in BG levels and intensity of insulin treatment. Corresponding to our observations in DM-0 rats, diabetes/hyperglycemia-induced reductions in Akt activity have been reported in murine retinal endothelial cells (Kondo et al. 2004), and in rat myocardium (Dobrzynski et al. 2002, Laviola et al. 2001). Considering established pathways of Akt activation, one of plausible explanations for reduced Akt activity in DM-0 is absolute lack of insulin. Indeed, Akt activity determined both as expression of P-Ser473-Akt and by kinase assay paralleled plasma insulin levels in diabetic rats.

Furthermore, hyperglycemia could also contribute. High glucose has been shown to be associated with reduced ability of Akt to be activated by known agonists in the skeletal muscle and endothelial cells (el-Remessy et al. 2005, Kondo and Kahn 2004, Krook et al. 1997). Indeed, similar phenomenon was observed in the present study. The same dose of insulin that only partially restored Akt activity in DM-4 rats, resulted in a significant increase in Akt phosphorylation in non-diabetic, normoglycemic animals. The ability of insulin to markedly enhance Akt phosphorylation in normal rats, as opposed to mere restoration of P-Akt expression in diabetic rats with insulin treatment that demonstrated similar, or in the case of DM-12 rats, even higher plasma insulin levels, suggests some degree of resistance to insulin actions in the diabetic kidney.

Interestingly, studies focusing on alterations of renal Akt signaling in the diabetic kidney and in renal cells in vitro exposed to high glucose have so far provided conflicting evidence. Our observations are in accordance with the report by Lin et al. (2006) showing markedly lower phospho-Akt expression in mesangial cells exposed to high glucose in conjunction with enhanced apoptosis. In contrast, growth hormone-induced Akt activation in STZ-diabetic rats was enhanced as compared to control animals (Thirone et al. 2002). Other studies have reported rapid glucose-induced Akt phosphorylation in murine and rat mesangial cells (Sheu et al. 2004), proximal tubular cells (Mariappan et al. 2007), glomerular epithelial cells, and enhanced renal Akt activity in STZ-diabetes in vivo (Lee et al. 2007).

We can only speculate about the explanation for disparate findings in the present studies and the above discussed evidence. Time factor may be important. For 

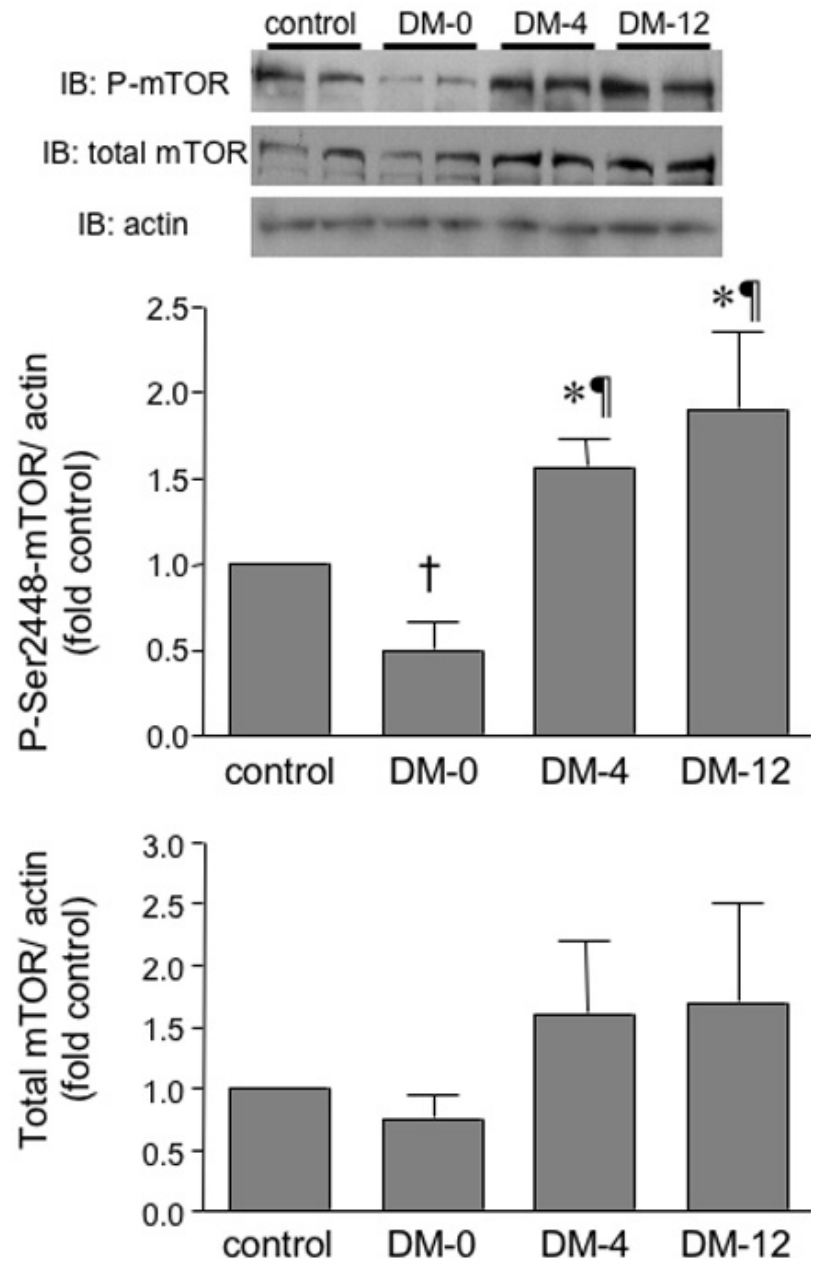

Fig. 2. Renal cortical protein expression of $m T O R$ in control and diabetic rats. P-Ser2448-mTOR (active) and total mTOR in renal cortical homogenates harvested from control animals, diabetic rats without insulin treatment (DM-0), and diabetic rats treated with 4 IU (DM-4) and 12 IU (DM-12) of insulin as described in Methods. The data are presented as mTOR/actin ratios. Representative blots are shown in the upper inset. $* p<0.05$ vs. control; $+p<0.01$ vs. control; ๆ $p<0.01$ vs. DM- 0 .

example, high glucose-induced Akt phosphorylation in proximal tubular cells (Mariappan et al. 2007) was detectable up to $15 \mathrm{~min}$, but returned to baseline after 30 min of exposure to high glucose. In vivo, renal cortical Akt activation was detected in diabetic rats early (3-4 days) after induction of diabetes (Lee et al. 2007). In the present studies, the kidneys were analyzed 4 weeks after the induction of diabetes. These differences in study designs indicate that present findings are not necessarily in disagreement with the above discussed renal evidence. High-glucose activation of Akt may be a phenomenon characteristic for acute phase of hyperglycemia/diabetes.

Out of large number of down-stream targets of Akt, mTOR was chosen for its role in initiation of protein translation (Kasinath et al. 2006, Scott et al. 1998) and possible involvement in enhanced renal production of
A.
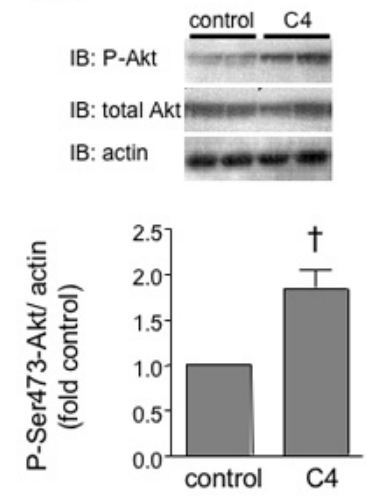

B.
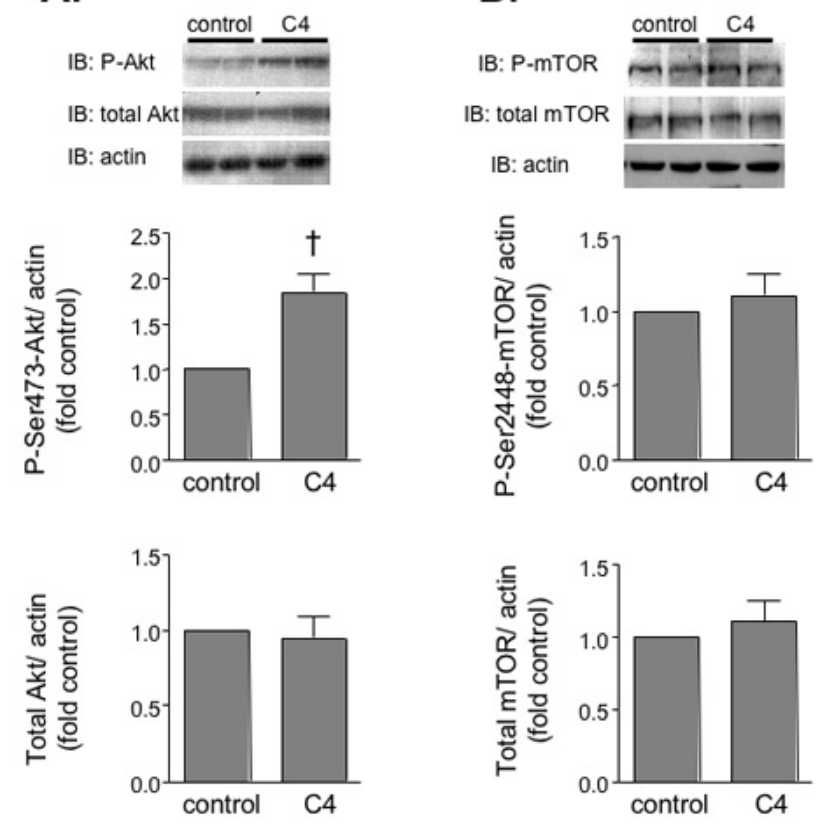

Fig. 3. Effects of insulin on Akt and mTOR in normal rats. Expression of P-Ser473-Akt (active) and total Akt (Panel A), and P-Ser2448-mTOR (active) and total mTOR (Panel B) in renal cortical homogenates harvested from control animals, and nondiabetic animals treated with $4 \mathrm{IU}$ of insulin was determined by western blotting. The data are presented as Akt/actin and mTOR/actin ratios. Representative blots are shown in the upper inset. $\dagger p<0.001$ vs. control.

ECM proteins (Senthil et al. 2003). In DM-0 rats, phosphorylation status of mTOR paralleled observed changes in indices characterizing Akt activity. However, diabetic rats with suboptimal and intensive insulin treatment demonstrated increases in mTOR phosphorylation as compared to control animals, and an opposite trend compared with DM-0. Moreover, in contrast to insulin-treated diabetic animals, there were no increases in mTOR phosphorylation in non-diabetic rats receiving insulin, at a dose sufficient to markedly activate Akt. These findings combined suggest that both hyperglycemia and insulin are required for renal mTOR activation in diabetes. In vitro, both high glucose and insulin have been recently shown to activate mTOR in murine proximal tubular cells, although the additive effect of glucose and insulin has not been reported (Mariappan et al. 2007).

Kidney/body weight ratios were markedly elevated in all groups of diabetic rats, although renal hypertrophy was attenuated by insulin treatment. This phenomenon indicates that signaling pathways leading to renal hypertrophy in severely diabetic rats lacking insulin treatment and in diabetic rats with moderate or mild hyperglycemia and insulin treatment may differ. 
Furthermore, mTOR phosphorylation did not parallel observed pattern of Akt activity in diabetic rats suggesting that in addition to Akt, some other signaling pathways contribute to activation of this molecule in diabetes. Indeed, Akt-independent pathways leading to mTOR phosphorylation have been recently suggested. S6 kinase 1 (S6K1) has been identified as direct mTOR kinase (Holz and Blenis 2005) acting down-stream from protein kinase C. Moreover, high-glucose-induced suppression of AMP-activated protein kinase, a natural inhibitor of mTOR activation, appears to be also operative in glomerular epithelial cells (Lee et al. 2007).

At this stage, it is impossible to determine the significance of alterations in renal Akt signaling for the development of kidney disease in Type 1 diabetes. Theoretically, Akt may be involved not only in protective signaling in the kidney (Shiojima and Walsh 2002), but it can also mediate deleterious effects such as renal hypertrophy. In contrast, mTOR activation seems to play, in accordance with its assumed role in enhanced production of ECM in the diabetic kidney, a role in the pathophysiology of diabetic nephropathy. Indeed, recent experimental study has demonstrated nephroprotective action of long-term mTOR inhibition with rapamycin (Lloberas et al. 2006).
In summary, diabetic rats with severe hyperglycemia, lack of insulin treatment and low plasma insulin levels, demonstrated reduced renal cortical Akt activity. This phenomenon was reversible by a tight metabolic control. Expression of active mTOR, one of down-stream effectors of Akt, was also reduced in severely diabetic animals. However, combination of hyperglycemia and insulin treatment resulted in increased mTOR phosphorylation. Chronic administration of insulin in normal rats lead to Akt, but not mTOR activation. These observations show that glycemic control and intensity of insulin treatment are important modulators of renal Akt and mTOR activity in diabetes and suggest that, in addition to Akt, other signaling pathways contribute to renal mTOR activity in diabetes.

\section{Conflict of Interest}

There is no conflict of interest.

\section{Acknowledgements}

These studies were supported by the institutional Grant MZO 00023001 of the Czech Ministry of Health Care. We are grateful to J. Vesela, H. Seidlova and I. Musilova for their excellent technical assistance.

\section{References}

BHANDARI BK, FELIERS D, DURAISAMY S, STEWART JL, GINGRAS AC, ABBOUD HE, CHOUDHURY GG, SONENBERG N,KASINATH BS: Insulin regulation of protein translation repressor 4E-BP1, an eIF4Ebinding protein, in renal epithelial cells. Kidney Int 59: 866-875, 2001.

COOPER ME: Pathogenesis, prevention, and treatment of diabetic nephropathy. Lancet 352: 213-219, 1998.

DOBRZYNSKI E, MONTANARI D, AGATA J, ZHU J, CHAO J, CHAO L: Adrenomedullin improves cardiac function and prevents renal damage in streptozotocin-induced diabetic rats. Am J Physiol 283: E1291-E1298, 2002.

DOWNWARD J: Mechanisms and consequences of activation of protein kinase B/Akt. Curr Opin Cell Biol 10: 262$267,1998$.

EL-REMESSY AB, BARTOLI M, PLATT DH, FULTON D, CALDWELL RB: Oxidative stress inactivates VEGF survival signaling in retinal endothelial cells via PI 3-kinase tyrosine nitration. J Cell Sci 118: 243-252, 2005.

HOLZ MK, BLENIS J: Identification of S6 kinase 1 as a novel mammalian target of rapamycin (mTOR)phosphorylating kinase. $J$ Biol Chem 280: 26089-26093, 2005.

KASINATH BS, MARIAPPAN MM, SATARANATARAJAN K, LEE MJ, FELIERS D: mRNA translation: unexplored territory in renal science. J Am Soc Nephrol 17: 3281-3292, 2006.

KOMERS R, LINDSLEY JN, OYAMA TT, COHEN DM, ANDERSON S: Renal p38 MAP kinase activity in experimental diabetes. Lab Invest 87: 548-558, 2007.

KONDO T, KAHN CR: Altered insulin signaling in retinal tissue in diabetic states. J Biol Chem 279: 37997-38006, 2004. 
KROOK A, KAWANO Y, SONG XM, EFENDIC S, ROTH RA, WALLBERG-HENRIKSSON H, ZIERATH JR: Improved glucose tolerance restores insulin-stimulated Akt kinase activity and glucose transport in skeletal muscle from diabetic Goto-Kakizaki rats. Diabetes 46: 2110-2114, 1997.

LAVIOLA L, BELSANTI G, DAVALLI AM, NAPOLI R, PERRINI S, WEIR GC, GIORGINO R, GIORGINO F: Effects of streptozocin diabetes and diabetes treatment by islet transplantation on in vivo insulin signaling in rat heart. Diabetes 50: 2709-2720, 2001.

LEE MJ, FELIERS D, MARIAPPAN MM, SATARANATARAJAN K, MAHIMAINATHAN L, MUSI N, FORETZ M, VIOLLET B, WEINBERG JM, CHOUDHURY GG, KASINATH BS: A role for AMP-activated protein kinase in diabetes-induced renal hypertrophy. Am J Physiol 292: F617-F627, 2007.

LIN CL, WANG JY, HUANG YT, KUO YH, SURENDRAN K, WANG FS: Wnt/beta-catenin signaling modulates survival of high glucose-stressed mesangial cells. J Am Soc Nephrol 17: 2812-2820, 2006.

LLOBERAS N, CRUZADO JM, FRANQUESA M, HERRERO-FRESNEDA I, TORRAS J, ALPEROVICH G, RAMA I, VIDAL A, GRINYO JM: Mammalian target of rapamycin pathway blockade slows progression of diabetic kidney disease in rats. J Am Soc Nephrol 17: 1395-1404, 2006.

MARIAPPAN MM, FELIERS D, MUMMIDI S, CHOUDHURY GG, KASINATH BS: High glucose, high insulin, and their combination rapidly induce laminin-betal synthesis by regulation of mRNA translation in renal epithelial cells. Diabetes 56: 476-485, 2007.

SCOTT PH, BRUNN GJ, KOHN AD, ROTH RA, LAWRENCE JC, JR.: Evidence of insulin-stimulated phosphorylation and activation of the mammalian target of rapamycin mediated by a protein kinase $\mathrm{B}$ signaling pathway. Proc Natl Acad Sci USA 95: 7772-7777, 1998.

SENTHIL D, CHOUDHURY GG, MCLAURIN C, KASINATH BS: Vascular endothelial growth factor induces protein synthesis in renal epithelial cells: a potential role in diabetic nephropathy. Kidney Int 64: 468-479, 2003.

SHEU ML, HO FM, CHAO KF, KUO ML, LIU SH: Activation of phosphoinositide 3-kinase in response to high glucose leads to regulation of reactive oxygen species-related nuclear factor- $\kappa \mathrm{B}$ activation and cyclooxygenase-2 expression in mesangial cells. Mol Pharmacol 66: 187-196, 2004.

SHIOJIMA I, WALSH K: Role of Akt signaling in vascular homeostasis and angiogenesis. Circ Res 90: 1243-1250, 2002.

THIRONE AC, SCARLETT JA, GASPARETTI AL, ARAUJO EP, LIMA MH, CARVALHO CR, VELLOSO LA, SAAD MJ: Modulation of growth hormone signal transduction in kidneys of streptozotocin-induced diabetic animals: effect of a growth hormone receptor antagonist. Diabetes 51: 2270-2281, 2002. 